\title{
ELISA for Bovine Interleukin-1 Receptor Antagonist and Its Application to Mastitic Sera and Whey
}

\author{
Hitoki YAMANAKA, Keiichi HISAEDA ${ }^{1}$, Katsuro HAGIWARA, Rikio KIRISAWA and Hiroshi IWAI* \\ Veterinary Microbiology, School of Veterinary Medicine, Rakuno Gakuen University, 582 Bunkyodai Midorimachi, Ebetsu, Hokkaido 069_ \\ 8501, and ${ }^{1)}$ Doi Veterinary Clinic Center, Ehime Prefectural Federation of Agricultural Mutual Association, Doi, Ehime 799-0700, Japan
}

(Received 24 November 1999/Accepted 18 February 2000)

ABSTRACT. A sandwich ELISA for the bovine IL-1 receptor antagonist (bIL-1ra) was developed using recombinant (r) bIL-1 ra produced by Escherichia coli, anti-rbIL-1ra rabbit IgG, its biotinylated one and avidin-peroxidase. This ELISA system enabled detection of rbIL-1ra at a concentration of more than $2 \mathrm{ng} / \mathrm{ml}$. This ELISA was applied to quantitation of bIL-1 ra in sera and whey of mastitic and healthy cows. The results indicate that although IL-1ra levels in healthy and mastitic sera and whey were comparable, serum IL-1ra/IL- $1 \beta$ ratio of euthanized cows was significantly lower than that of the recovered.-KEY WORDS: bovine, ELISA, IL-1ra.

J. Vet. Med. Sci. 62(6): 661-664, 2000

Interleukin-1 receptor antagonist (IL-1 ra) is a protein that binds to the IL-1 receptor (IL-1R) without inducing a signal of its own and blocks the binding of IL- $1 \alpha$ and IL- $1 \beta$ $[1,3-5,8]$. Therefore, IL-1 ra is considered to modify inflammation mediated by IL-1 as well as the soluble IL-1 receptor (sIL-1R) [4]. Determination of IL-1, sIL-1R and IL-1ra levels in clinical specimens is considered to be useful for evaluating a disease condition.

We previously reported the sequence of bovine (b) IL-1ra and production of recombinant (r) IL-1 ra in E. coli [9]. In the present paper, we describe a sandwich ELISA for bIL1ra using anti-rbIL-1 ra rabbit IgG and application of the assay to bovine sera and whey with or without mastitis.

Production and purification of rbIL-1 ra was performed as described previously [9]. Molecular weight of rbIL-1 ra was determined by $12.5 \%$ sodium dodecyl sulfate (SDS)polyacrylamide gel electrophoresis (PAGE) according to the method of Laemmli [10]. Other recombinant bovine cytokines used in this study were IL- $1 \alpha$, IL- $1 \beta$, IL-6, IFN- $\gamma$ and TNF- $\alpha$, which were prepared fundamentally according to the method described previously [7].

The biological activity of rbIL-1 ra was determined by suppression of A375 cell growth inhibition activity of bovine IL-1 [6, 11] as described previously [9].

Antibodies to cytokines, including IL-1ra, were prepared according to the methods described previously [7]. Specific rabbit anti-rbIL-1ra IgG was purified from the ammonium sulfate precipitation fraction of immune sera by affinity chromatography using cyanogen bromide-activated Sepharose 4B (Amersham Pharmacia Biotech, U.K.) coupled with rbIL-1ra. The purified IgG was used as a capture antibody for IL-1ra without any modification and as a detection antibody after biotinization using a commercially available kit (Amersham Pharmacia, U.K.).

The specificity of purified rabbit anti-rbIL-1ra IgG was

* CoRrespondence to: IWAi, H., Veterinary Microbiology, School of Veterinary Medicine, Rakuno Gakuen University, 582 Bunkyodai Midorimachi, Ebetsu 069-8501, Japan. confirmed by Western blotting as described previously [6].

For the development of a sandwich ELISA for bIL-1ra, an EIA/RIA plate (Corning Costar Co., Tokyo, Japan) was coated with $50 \mu l$ of purified rabbit anti-rbIL-1ra IgG that was diluted appropriately with $0.05 \mathrm{M}$ carbonate buffer (pH9.6) and incubated overnight at $4{ }^{\circ} \mathrm{C}$. The wells were washed 3 times with PBS containing $0.05 \%$ tween-20 (TPBS), filled with T-PBS containing 25\% Block Ace (Dainihonseiyaku Co., Tokyo, Japan), and then kept at $37^{\circ} \mathrm{C}$ for $1 \mathrm{hr}$ to block non-specific absorbance. After 3 washings with T-PBS, serum, whey or serially diluted rbIL1ra was added to the wells, and the plate was incubated for $2 \mathrm{hr}$ at room temperature. Then, after 6 washings with TPBS, appropriately diluted biotinylated anti-rbIL-1 ra rabbit IgG was added to the wells and incubated for $1 \mathrm{hr}$ at room temperature. After 6 washings, appropriately diluted streptavidin horseradish peroxidase conjugate (Amersham Pharmacia Biotech, U.K.) was added to the wells and incubated for an additional $1 \mathrm{hr}$ at room temperature. After 6 washings with T-PBS, $100 \mu l$ of substrate solution $(2 \mathrm{mM}$ 2,2'-Azino-bis(3-Ethylbenzthiazoline-6-sulfonic acid) and $0.004 \% \mathrm{H}_{2} \mathrm{O}_{2}$ in $50 \mathrm{mM}$ citrate buffer, $\mathrm{pH} 4.0$ ) was added to each well and left for $1 \mathrm{hr}$ at room temperature to detect bound enzymes that produced color, and the intensity of the colored product was measured by a microplate reader (Nihon Intermed Co., Japan) at $405 \mathrm{~nm}$. The concentration of IL-1 ra in samples was determined from the plotted standard curve. Detection of bIL- $1 \beta$ by ELISA was performed as described previously [7].

Bovine serum and whey were collected from acute Gram-negative bacterial mastitic Holstein cattle $(n=28,8$ cows, from parturition to 5 days; 14 cows, from 6 to 90 days; 5 cows, from 91 to 210 days; a cow, more than 211 days) on days $0,3,7,14$ of illness and from clinically normal Holstein cattle $(n=30$, a cow, from parturition to 5 days; 6 cows, from 6 to 90 days; 12 cows, from 91 to 210 days; 11 cows, more than 211 days). Causative agents of the mastitis in the cattle were E. coli $(\mathrm{n}=11)$, Klebsiella 


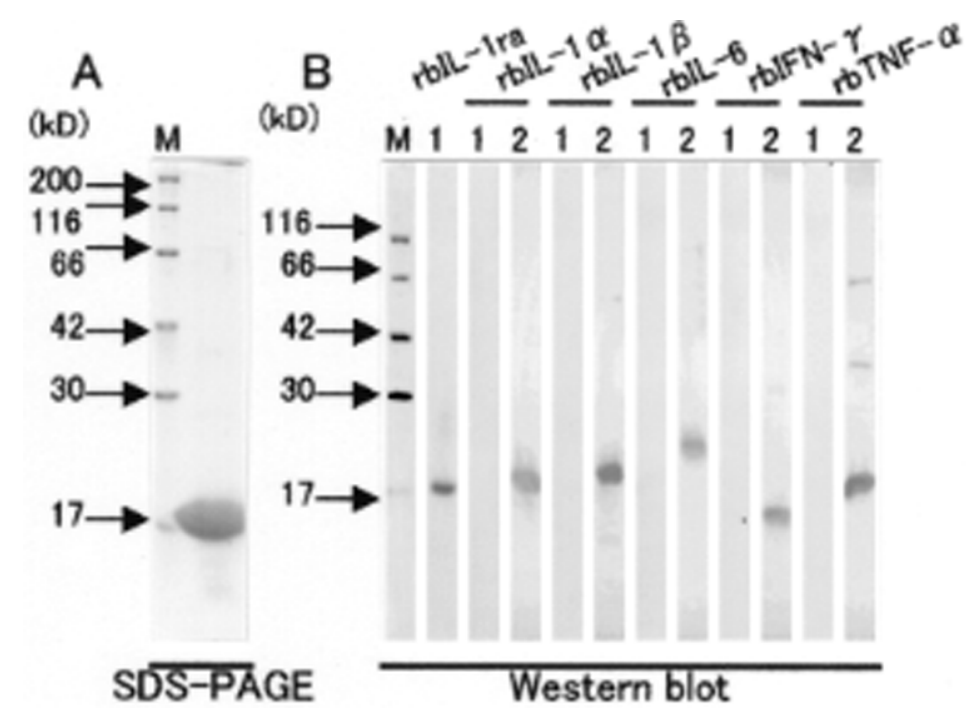

Fig. 1. Molecular weight of purified rbIL-1ra as determined by SDS-PAGE (A) and reactivity of purified polyclonal anti-rbIL-1ra antibody with rbIL-1ra, IL$1 \alpha$, IL- $1 \beta$, IL- 6 , IFN- $\gamma$ and TNF- $\alpha$ as determined by Western blotting (B). Lane 1, probed with anti-rbIL-1ra rabbit IgG; Lane 2, probed with polyclonal antibodies against corresponding cytokines.

pneumoniae $(\mathrm{n}=16)$ and Proteus sp. $(\mathrm{n}=1)$. The mastitic cattle were treated with antibiotics to which the causative agent was susceptible, and all of the cows, except for 4 cows that were euthanized due to a poor prognosis, recovered by day 14 after the onset of mastitis.

To demonstrate IL-1ra in bovine serum in addition to the ELISA, immunoprecipitation was performed using cyanogen bromide-activated Sepharose 4B (Amersham Pharmacia Biotech, U.K.) coupled with anti-rbIL-1 ra rabbit IgG according to the method described previously [7]. The condition of adsorptive reaction were overnight at $4{ }^{\circ} \mathrm{C}$ with IL-1ra-positive in serum sample by sandwich ELISA and after washing with PBS, adsorbed gel was electrophoresed in SDS-polyacrylamide gel.

Statistical significance was evaluated by Student's $t$-test and a $P$ value $<0.05$ was considered statistically significant.

The molecular weight of purified rbIL-1ra was confirmed by SDS-PAGE to be about $17 \mathrm{kD}$ (Fig. 1A), which coincided with those in previous reports on human IL-1ra [1-4]. In previous report, we reported that the rbIL1 ra inhibited IL- $1 \beta$ activity on A375 cells in a dosedependent manner [9]. In the present study, we confirmed that rbIL-1ra also inhibited IL- $1 \alpha$ activity (data not shown), indicating that rbIL-1ra is biologically active. Specificity of anti-rbIL-1ra rabbit IgG was demonstrated by Western blot analysis, showing single band positioning of about 17 $\mathrm{kD}$ on rbIL-1ra but not on the other cytokines tested (Fig. 1B). As shown in Fig. 2, the present sandwich ELISA for IL-1 ra enabled detection of rbIL-1 ra at a concentration of more than $2 \mathrm{ng} / \mathrm{m} l$. To improve the sensitivity of the ELISA, it is essential to establish hybridomas secreting monoclonal antibodies with higher reactivity.

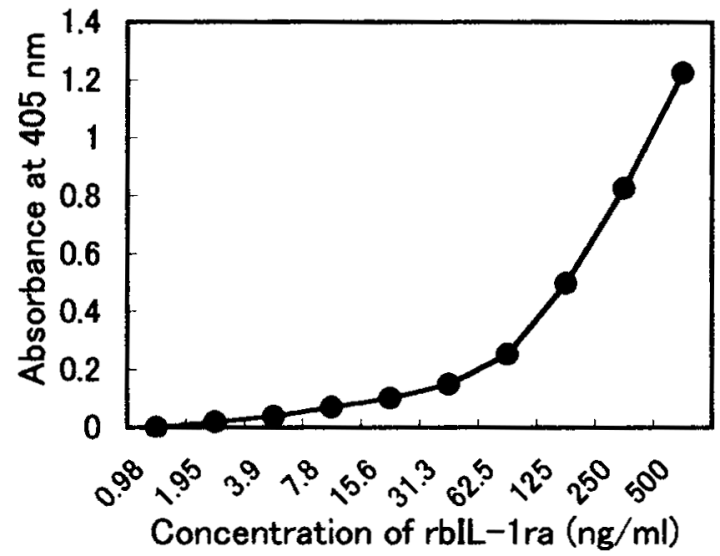

Fig. 2. Detection of rbIL-1ra by sandwich ELISA. The wells were coated with diluted rabbit anti-rbIL-1 ra IgG. Serially 2 fold diluted rbIL-1ra was applied to the coated wells, and then diluted biotinylated rabbit antirbIL-1ra IgG were added to the wells.

We applied this method to quantitation of IL-1ra in sera and whey of healthy and mastitic cows. We also measured IL- $1 \beta$ levels in the same samples. As shown in Fig. 3, IL1 ra was detected in sera and whey of both healthy and mastitic cows, and the levels in sera were higher than those in whey. The mean IL-1ra concentration in serum samples from the mastitic cows, except for the serum samples obtained on day 14 , was not significantly different from that in the serum samples from healthy cows. We tried to detect IL-1 ra in serum, of which concentration was more than $3,000 \mathrm{ng} / \mathrm{m} l$, by immunoprecipitation. A specific band 

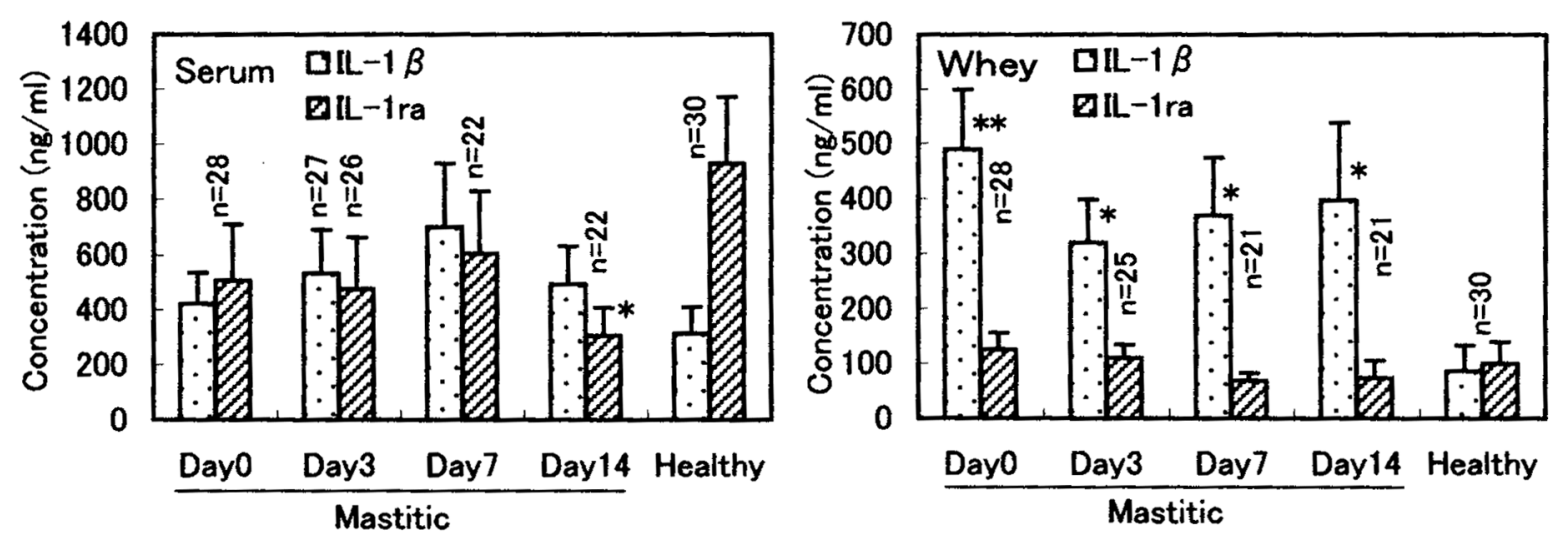

Fig. 3. IL-1 $\beta$ and IL-1 ra concentrations in sera and whey of mastitic cows during a 2-week period after the onset of mastitis and of healthy cows. Data are expressed as mean concentration with S.E. $* \mathrm{P}<0.05$, $* * \mathrm{P}<0.01$, Asterisk represents statistical significance when compared with the concentration in serum or whey of healthy cows.

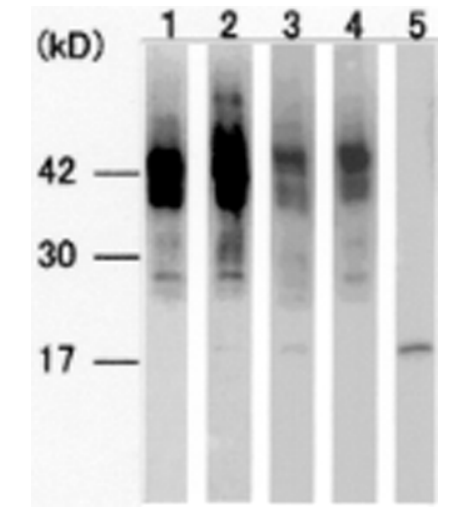

Fig. 4. Detection of IL-1ra in serum of mastitic cows by immunoprecipitation. Lane 1, unadsorbed gel; 2, gel reacted with IL-1ra-positive serum sample (more than $3,000 \mathrm{ng} / \mathrm{m} l$ by ELISA); 3 , gel reacted with rbIL-1 ra $(10 \mu \mathrm{g} / \mathrm{ml}) ; 4$, gel reacted with IL-1ra-negative serum sample; 5 , rbIL-1ra $(10 \mu \mathrm{g} / \mathrm{m} l)$.

positioning of about $17 \mathrm{kD}$ could be detected (see Fig. 4).

The mean concentration of IL- $1 \beta$ in serum samples from the mastitic cows were also not different from that in serum samples of the healthy cows. The reason for this small difference between serum IL- $1 \beta$ concentration in healthy and mastitic cows may be the presence of IL- $1 \beta$ in sera of healthy pregnant cattle [7]. In the case, since production of IL-1 ra follows the production of IL-1 [1-4], the small difference between IL-1 ra concentration in healthy and mastitic sera may also reflect the presence IL-1 in pregnant cattle. The mean concentration of IL- $1 \beta$ in whey of mastitic cows was significantly higher than that in healthy cows throughout the observation period.

IL- $1 \beta$ levels of healthy cattle in the present study was markedly higher than those in previous report [7]. The reason of such differences was not clear, however, they may

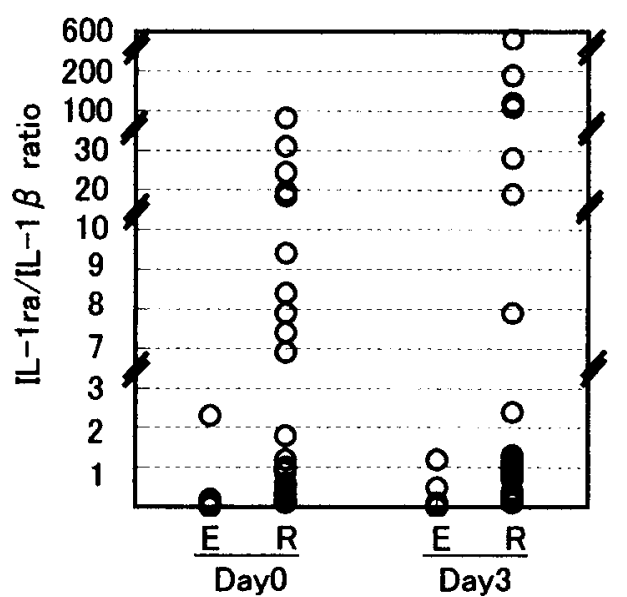

Fig. 5. IL-1ra/IL-1 $\beta$ ratio of sera from the euthanized $(\mathrm{E})$ or recovered $(\mathrm{R})$ mastitic cows, during a period of 3 days after the onset of the disease.

be regional difference between Hokkaido and Ehime, because sera obtained from healthy cattle recently in Hokkaido also showed lower value.

The results were rearranged according to the consequence of mastitic cows, i. e., recovered or euthanized, indicated that in the euthanized group (data on day 0 and day 3 are shown because samples were not available on day 7 or later), the mean serum IL-1ra concentration was lower than that in the recovered group, and that of IL- $1 \beta$ were higher (data not shown). Therefore, IL- $1 \mathrm{ra} / \mathrm{IL}-1 \beta$ ratio was compared between the groups (see Fig. 5). The ratio of the euthanized group was significantly lower than that of the recovered on day $0(\mathrm{p}<0.05)$. This indicate combination assay for both IL-1ra and IL- $1 \beta$ may be useful to evaluate inflammatory condition.

We examined IL-1 inhibition activity of mastitic sera by 
A375 cell growth inhibition tests, but no correlation was found between IL-1 ra concentration and IL-1 inhibition activity (data not shown), indicating the presence of other inhibitory factors, such as sIL-1R.

In conclusion, although a polyclonal antibody was used, the sandwich ELISA for bIL-1ra described here enabled detection of the antagonist in clinical specimens.

This work was partially supported by a Grant-in-Aid of Gakujutsu Frontier Cooperative Research in Rakuno Gakuen University.

\section{REFERENCES}

1. Arend, W. P. 1993. Adv. Immunol. 54: 167-227.

2. Arend, W. P., Smith, M.F. JR., Janson, R.W. and Joslin, F.G. 1991. J. Immunol. 147: 1530-1536.

3. Dinarello, C.A. 1991. Blood 77: 1627-1652.

4. Dinarello, C.A. 1996. Blood 87: 2095-2147.
5. Dinarello, C.A. and Thompson, R.C. 1991. Immunol. Today. 12: 404-410

6. Goto, M., Kirisawa, R., Tajima, M., Takahashi, K. and Iwai, H. 1995. J. Vet. Med. Sci. 57: 523-525.

7. Goto, M., Maruyama, M., Kitadate, K., Kirisawa, R., Obata, Y., Koiwa, M. and Iwai, H. 1997. J. Vet. Med. Sci. 59: 437441.

8. Hannum, C.H., Wilcox, C.J., Arend, W.P., Joslin, F.G., Dripps, D.J., Heimdal, P.L., Armes, L.G., Sommer, A., Eisenberg, S.P. and Thompson, R.C. 1990. Nature (Lond.) 343: 336-340.

9. Kirisawa, R., Fukuda, T., Yamanaka, H., Hagiwara, K., Goto, M., Obata, Y., Yoshino, T. and Iwai, H. 1998. Vet. Immunol. Immunopathol. 62: 197-208.

10. Laemmli, U.K. 1970. Nature (Lond.) 227: 680-685.

11. Nakai, S., Mizuno, K., Kaneda, M. and Hirai, Y. 1988. Biochem. Biophys. Res. Commun. 154: 1189-1196.

12. Poutsiaka, D.D., Clark, B.D., Vannier, E. and Dinarello, C.A. 1991. Blood 78: 1275-1281. 